\title{
Patterns of Failure and Need for Biliary Intervention in Resected Biliary Tract Cancers After Chemoradiation
}

\author{
Rebecca M. Shulman, MD, and Joshua E. Meyer, MD \\ Department of Radiation Oncology, Fox Chase Cancer Center, Philadelphia, PA
}

Biliary tract cancers (BTCs) are adenocarcinomas that may occur in the gallbladder, the intrahepatic bile ducts, or any segment of the extrahepatic bile ducts from the hilum to the ampulla. All BTCs are aggressive and rare, accounting for only $4 \%$ of gastrointestinal malignancies, although their incidence is increasing everywhere for reasons that are mysterious. ${ }^{1}$ Radical resection is considered the only possibility of cure, but dissemination of BTC from the primary site, commonly into the porta hepatis, restricts curative surgical intervention to approximately one-third of patients. ${ }^{2}$ Even after surgery, the prognosis of BTC is poor, particularly for those with positive resection margins (R1) or involvement of regional lymph nodes, with subgroups making up $40-50 \%$ of patients. The 5-year survival for resectable BTCs is $20-40 \%{ }^{3}$ Recurrence after surgery is seen in up to $68 \%$ of patients at 2 years; in one series of 177 patients, first recurrences were locoregional in only $15 \%$ of those with gallbladder cancer (GBC) and in 59\% of those with cholangiocarcinoma (CCA). ${ }^{4}$

In the article entitled 'Patterns of Failure and Need for Biliary Intervention in Resected Biliary Tract Cancers After Chemoradiation', ${ }^{5}$ Dee and colleagues report a retrospective study of all patients with BTC who were treated with curative surgery and adjuvant radiotherapy (RT) at a single institution over a 10 -year period. RT was routinely administered in their center for BTC patients at high risk, and was usually accompanied by chemotherapy (CT). The authors provide a thorough summary of their results,

(C) Society of Surgical Oncology 2020

First Received: 27 July 2020

Accepted: 3 August 2020;

Published Online: 17 August 2020

J. E. Meyer, MD

e-mail: Joshua.meyer@fccc.edu assembling diverse types of data in an attempt to identify the predictors and complications of locoregional failure (LRF) after treatment. Two-year LRF was $23.5 \%$ for the entire group of 80 patients, consistent with other reports. ${ }^{6}$ The patterns of failure recorded were the association of LRF with regional lymph node involvement (but not R1 resections) and the inability to predict LRF from the cancer site, the presence of genetic mutations, or the stage of tumor. LRF was often followed by the need for instrumentation of the biliary tract, a major source of morbidity after surgery. Indeed, local complications proved fatal for one-third of patients who developed them. No data regarding distant metastases are provided, an omission that reflects the authors' decision to focus on local recurrences, perhaps for their direct bearing on the potential role of adjuvant RT. In this context, it is useful to note that the biliary strictures they observed were far more likely to have been malignant than radiation-induced. ${ }^{7}$

Do the reported patterns of LRF, as the authors suggest, provide a clue that adjuvant chemoradiation (CRT) for BTC improves outcomes? A 50-year history of retrospective institutional series, registry studies, and meta-analyses has studied this question, beginning, in 1977, with Kopelson et al., ${ }^{8}$ who reported that three patients at the Massachusetts General Hospital with BTC who had received adjuvant RT after surgery survived 32 months, 19 months longer than those who received surgery alone.

Dozens of similar, although usually larger, retrospective studies have since been reported. Many of those studies demonstrated a clinical benefit for adjuvant therapy using RT alone, ${ }^{9}$ CT alone, ${ }^{10}$ or a combination of both. ${ }^{11}$ Weighing against such favorable studies were a smaller number of similar studies reporting no benefit for adjuvant therapy. ${ }^{4,12}$ However, more troublesome than the negative reports were the inconsistencies among the positive reports themselves. Differences in cancer site, stage and grade of 
tumor, surgical and adjuvant treatments, clinical endpoints, and the selection criteria for patients often made direct comparisons impossible. To this difficulty was added the statistical challenge of small patient subgroups and the shortcomings of the retrospective design, vulnerable by its very nature to selection bias and gaps in the retrieval of significant patient and treatment data.

The inability of retrospective studies to put to rest questions of cause and effect has produced an unsatisfactory status quo: prevailing expert opinion holds that the value of adjuvant therapy in BTC is 'not well-defined', ${ }^{13}$ while $70 \%$ of cancer centers worldwide-noting a $68 \%$ 2-year relapse rate after surgery-use RT/CT routinely for high-risk patients. ${ }^{2}$ Supporting this practice is the quite tolerable $2-9 \%$ incidence rate of late radiation-induced toxicity (chiefly obstruction and bleeding). ${ }^{14}$ With this history as background, Dee et al. claim that the patterns of LRF they meticulously record add to the evidence of previous retrospective studies and suggest a clinical benefit for adjuvant CRT. Because theirs is not a controlled study, the claim rests on the unexpected finding that LRF occurred in $\mathrm{R} 0$ and R1 resections with equal frequency. Since earlier studies have reported a relatively poor prognosis for patients after R1 resection, ${ }^{14}$ adjuvant RT may have benefited such patients selectively. However, while it is true that the association of $\mathrm{R} 1$ resections and early recurrence has been reported often, it has not been reported consistently. In one recent study, ${ }^{15}$ for example, LRF after surgical resection for CCA was unrelated to reported surgical margins, a result the authors attribute to a lack of statistical power but which may also reflect the difficulty of deciding when dysplasia denotes a positive margin. The authors' argument continues in logical fashion: if CRT explains the unexpectedly low rate of LRF after R1 resections, then the low rate of LRF might explain the improved survival of such patients reported elsewhere. However, this line of thinking evades the decreased survival of such patients in their own study. The inescapable problem, as the authors acknowledge, is that only the most tentative conclusions regarding the efficacy of CRT are permitted by the design of their study.

In recent years, great effort has been invested in multicenter and multinational studies addressing the efficacy of adjuvant CRT more directly. The SWOG 0809 study $^{6}$ of GBC and extrahepatic CCA, employing an adjuvant protocol of gemcitabine/capecitabine followed by CRT, achieved a particular milestone. Although it lacked a control group, the study's rigor, prospective design, sizable sample, uniformity of treatment protocol, and modest reported treatment toxicity exercised considerable influence on clinical practice. The 2-year overall survival rate of $65 \%$ after adjuvant therapy reported in SWOG 0809 was also noteworthy, exceeding historical standards by a large margin. To date, no study has provided a more compelling case that adjuvant CRT has a role in the treatment of resectable BTC.

Many phase III studies of GBC and extrahepatic CCA have now been completed or are underway, all of them adopting protocols of adjuvant CT to the exclusion of RT. The BCAT trial ${ }^{16}$ in Japan (gemcitabine) and the PRODIGE 12-ACCORD 18 trial $^{17}$ in France (gemcitabine/ oxaliplatin) found no benefit for such adjuvant therapy. These negative results contrast with the recent report of the British BILCAP trial, ${ }^{18}$ which randomized 447 postoperative patients with resected BTC to either 24 weeks of oral capecitabine or observation. The original intent-to-treat design proved to be underpowered for the unexpectedly high 2-year survival rate $(60 \%)$ of the observation group. However, a per-protocol analysis demonstrated a survival advantage for capecitabine, and a decreased rate of relapse for 2 years, but not beyond. Recent American Society of Clinical Oncology (ASCO) guidelines, ${ }^{19}$ recognizing the qualified efficacy of capecitabine, as well as its low cost, low toxicity, and availability for oral administration, identify capecitabine as the standard of care for resected BTC, while CRT retains its role as a discretionary option for patients with positive surgical margins or regional lymph node involvement.

The randomized, controlled studies of BTC performed in centers worldwide following SWOG 0809 have adopted variations of $\mathrm{CT}$ protocols previously demonstrating efficacy in unresectable or metastatic BTC. This is an understandable choice but it means that two kinds of technical progress have not yet received systematic study. The first of these is the development of novel forms of cancer treatment. Neoadjuvant RT is a particularly promising example that offers as potential advantages the reduction of tumor seeding during surgery, the sparing of surgical anastomoses, and the preservation of the vasculature (and oxygen supply) of radiated tissues. Support for a neoadjuvant RT approach to BTC is provided by favorable experience with RT in unresectable intrahepatic CCA. In that setting, tumor control can be dramatically improved with little toxicity if large ablative radiation doses are administered, with the precision made possible by intensity-modulated RT, respiratory gating, and other contemporary techniques. ${ }^{20}$

This introduction of technologically superior forms of adjuvant therapy has been accompanied by progress of a second kind; recent studies have demonstrated that we are moving toward replacement of the traditional classification of BTC based on anatomical site, by a classification scheme based on molecular subtypes. Investigations of altered signaling pathways and genetic mutations, including mutations affecting the expression of $\beta$-catenin ${ }^{21}$ and isocitrate dehydrogenase (IDH), ${ }^{22}$ have strongly implicated 
these alterations in biliary tract carcinogenesis. Such discoveries have direct implications for new therapeutic strategies. The management of BTC is thus entering an era in which technical advances in the delivery of established forms of treatment are occurring in parallel with the introduction of highly sophisticated, clinically relevant biological assays. Progress in managing this rare and lethal disease will depend on a readiness to test refinements in adjuvant therapies when they appear promising, and the ingenuity to draw upon our growing understanding of the molecular basis of treatment outcomes.

FUNDING The funding was provided by National Cancer Institute (Grant No. P30 CA006927).

DISCLOSURES Rebecca M. Shulman and Joshua E. Meyer declare no conflict of interest.

\section{REFERENCES}

1. Murakami Y, Uemura K, Hayasidani Y, et al. Indication for postoperative adjuvant therapy in biliary carcinoma based on analysis of recurrence and survival after surgical resection. Dig Dis Sci. 2009;54:1360-64. https://doi.org/10.1007/s10620-008-0 492-7

2. Horgan AM, Amir E, Walter T, et al. Adjuvant therapy in the treatment of biliary tract cancer: a systematic review and metaanalysis. J Clin Oncol. 2012;30:1934-40.

3. Stein A, Arnold D, Bridgewater J, et al. Adjuvant chemotherapy with gemcitabine and cisplatin compared to observation after curative intent resection of cholangiocarcinoma and muscle invasive gallbladder carcinoma (ACTICCA-1 trial): a randomized, multidisciplinary, multinational phase III trial. $B M C$ Cancer. 2015;15:564-75.

4. Jarnagin WR, Ruo L, Little SA, et al. Patterns of initial disease recurrence after resection of gallbladder carcinoma and hilar cholangiocarcinoma: implications for adjuvant therapeutic strategies. Cancer. 2003;98(8):1689-1700.

5. Dee E, Freret M, Morick N, et al. Patterns of failure and need for biliary intervention in resected biliary tract cancers after chemoradiation. Ann Surg Oncol. 2020. https://doi.org/10.1245/ s10434-020-08967-9.

6. Ben-Josef E, Guthrie KA, El-Khoueiry AB, et al. SWOG S0809: a phase II intergroup trial of adjuvant capecitabine and gemcitabine followed by radiotherapy and concurrent capecitabine in extrahepatic cholangiocarcinoma and gallbladder carcinoma. $J$ Clin Oncol. 2015;33(24):1617-24.

7. Nakakubo $\mathrm{Y}$, Kondo $\mathrm{S}$, Katoh $\mathrm{H}$, et al. Biliary stricture as a possible late complication of radiation therapy. Hepatogastroenterology. 2000;47;1531-32.

8. Kopelson G, Harisiadis L, Tretter P, et al. The role of radiation therapy in cancer of the extra-hepatic biliary system: an analysis of thirteen patients and a review of the literature of the effectiveness of surgery, chemotherapy and radiotherapy. Int J Radiat Oncol Biol Phys. 1977;2:883-94.

9. Gerhards MF, van Gulik TM, Gonzalez Gonzalez D, et al. Results of postoperative radiotherapy for resectable hilar cholangiocarcinoma. World J Surg. 2003;27:173-9.

10. Nakamura M, Nakashima $H$, Abe $T$, et al. Gemcitabine-based adjuvant chemotherapy for patients with advanced gallbladder cancer. Anticancer Res. 2014;34(6):3125-9.

11. Hoehn RS, Wima K, Ertel AE, et al. Adjuvant therapy for gallbladder cancer: an analysis of the National Cancer Data Base. $J$ Gastrointest Surg. 2015;19:1794-1801.

12. Pitt HA, Nakeeb A, Abrams RA, et al. Postoperative radiotherapy does not improve survival. Ann Surg. 1995; 221:788-97.

13. Valle JW, Borbath I, Khan SA et al. Biliary cancer: ESMO clinical practice guidelines for diagnosis, treatment and followup. Ann Oncol. 2016;29 Suppl 5:v28-v37.

14. Beltran MB, Allal AS, Gich I, et al. Is adjuvant radiotherapy needed after curative resection of extrahepatic biliary tract cancers? A systematic review with a meta-analysis of observational studies. Cancer Treat Rev. 2012;38:111-19.

15. Ghiassi-Nejad Z, Tarchi P, Moshier E, et al. Prognostic factors and patterns of locoregional failure after surgical resection in patients with cholangiocarcinoma without adjuvant radiation therapy: optimal field design for adjuvant radiation therapy. Int $J$ Radiat Oncol Biol Phys. 2017;99(4):805-11.

16. Ebata T, Hirano S, Konishi M, et al. Randomized clinical trial of adjuvant gemcitabine chemotherapy versus observation in resected bile duct cancer. Br J Surg. 2018;105(3):192-202.

17. Edeline J, Benabdelghani M, Bertaut A, et al. Gemcitabine and oxaliplatin chemotherapy or surveillance in resected biliary tract cancer (PRODIGE 12-ACCORD 18-UNICANCER GI): a randomized phase III study. J Clin Oncol. 2019;37:658-67.

18. Primrose JN, Fox RP, Palmer DH, et al. Capecitabine compared with observation in resected biliary tract cancer (BILCAP): a randomized, controlled, multicentre phase 3 study. Lancet Oncol. 2019;20:663-73.

19. Shroff RT, Kennedy EB, Bachini M, et al. Adjuvant therapy for resected biliary tract cancer: ASCO clinical practice guidelines. $J$ Clin Oncol. 2019;37(12):1015-28.

20. Tao R, Krishnan S, Bhosale PR, et al. Ablative radiotherapy dose leads to substantial prolongation of survival in patients with inoperable intrahepatic cholangiocarcinoma: a retrospective dose analysis. J Clin Oncol. 2016;34(3):219.

21. Schiffman SC, Nowacki MR, Spencer L, et al. Molecular factors associated with recurrence and survival following hepatectomy in patients with intrahepatic cholangiocarcinoma: a guide to adjuvant clinical trials. J Surg Oncol. 2014;109:98-107.

22. Farshidar F, Zheng S, Gringas M-C, et al. Integrative genomic analysis of cholangiocarcinoma identifies distinct IDH-mutant molecular profiles. Cell Rep. 2017;18:2780-94.

Publisher's Note Springer Nature remains neutral with regard to jurisdictional claims in published maps and institutional affiliations. 